\title{
Percepción de los entrenadores de alto rendimiento de atletismo: caracterización de su perfil polifacético Perception of high performance track and field coaches: characterization of their multifaceted profile \\ Ángeles Filgueira Pérez \\ UNED Madrid (España)
}

Resumen. En el presente estudio se ha tratado de obtener una aproximación al perfil ideal del entrenador de alto rendimiento en atletismo. En el mundo del deporte de alta competición, el entrenador debe desarrollar funciones de maestro, técnico y líder, lo cual hace difícil delimitar sus competencias (conocimientos, habilidades y cualidades personales). Por tanto, la principal motivación de este trabajo es establecer el papel que debe desempeñar el entrenador para la preparación física, técnica, táctica, psicológica y moral del deportista de alto nivel. Para ello, se ha considerado como población objeto de estudio al conjunto de entrenadores de alto rendimiento que en el momento de la investigación estuviesen activo, ya que interesaba realizar este estudio desde su propia perspectiva. Los datos recogidos forman parte de una investigación más amplia, llevada a cabo mediante encuesta, para la que se diseñó un cuestionario de 78 preguntas en el que se abarcaban tres temas: el perfil del entrenador y del deportista, así como la figura del formador de entrenadores en el Practicum. En este trabajo, nos centramos en el primer tema, el análisis de la información obtenida nos permite concluir que la práctica totalidad de los entrenadores de atletismo consideran necesario dominar con precisión los aspectos técnico-metodológicos. Además, consideran que su ética profesional debe estar regida por los principios de autonomía y beneficencia, de modo que valores como la honestidad y la justicia deben primar en el desarrollo de sus funciones.

Palabras clave. entrenador de alto rendimiento, perfil, técnico, maestro, líder, atletismo, deportista, conocimientos.

Abstract. In the current study, we have tried to get an approximation of the ideal profile of high performance coaches in athletics. In the high-level sports world, the coach must develop the role of teacher, technician and leader, which makes it difficult to delimit his/her competencies (knowledge, skills and personal qualities). Therefore, the main motivation of this work is to define the role that the coach plays in relation to the physical, technical, tactical, psychological and moral preparation of elite athletes. Keeping this aim as an objective, we have considered as the target population the high performance coaches who are active at the time of the investigation, since we were interested in knowing their own perspective. The data that was collected is a part of a wider investigation, conducted by a survey, for which we designed a questionnaire of 78 questions divided on three topics: the profile of the coach and the athlete, as well as the figure of the coach educator in the Practicum. In this work, we focus on the first topic and the analysis of the information that we obtained allows us to conclude that almost all the track and field coaches find it necessary to dominate the technical and methodological aspects. They also consider that professional ethics must be governed by the principles of autonomy and care that values like honesty and justice must prevail in the performance of their work.

Keywords. high performance coach, profile, technical, teacher, leader, athletics, athlete, knowledge.

\section{Introducción}

El perfil del entrenador de alto rendimiento (EAR) ha sido estudiado en nuestro país desde tres perspectivas: la técnica, la educativa y la de liderazgo. En primer lugar, muchas publicaciones han estudiado desde la perspectiva técnica la figura del EAR (Ruiz \& Bañuelos, 1997; Del Villar \& Fuentes, 1999; Saneugenio \& Escontrela, 2000; Ortega, 2005). Esta perspectiva se basa en la eficacia, está justificada cuando establece su interés en los resultados deportivos alcanzados por el deportista bajo la dirección del entrenador. Es razonable ya que el deporte de alto nivel se considera de interés para el Estado por su función representativa del país en las pruebas o competiciones deportivas oficiales de carácter internacional, además de que constituye un factor esencial en el desarrollo deportivo, por el estímulo que supone para el fomento del deporte. Así pues, mientras que la figura del entrenador de alto rendimiento no está legislada, ya que ningún decreto estatal lo contempla, no pasa lo mismo con el deportista de alto nivel. Aéste se le exige un rendimiento y clasificación entre los mejores a nivel nacional e internacional. Esta exigencia debe adaptarse a unos criterios selectivos para poder ser reconocido y acceder a unas medidas de apoyo deportivo. No obstante, estas medidas dependen de los resultados deportivos y son limitadas en el tiempo.

El objetivo prioritario de la alta competición es la búsqueda del máximo rendimiento del deportista mediante la práctica. Éste aprende unas técnicas facilitadas por su entrenador. Es precisamente el hecho de que el resultado óptimo se vincule a la idoneidad de la metodología de enseñanza lo que define al modelo técnico educativo. Dicho en otras palabras, el modelo técnico se centra en la enseñanza de las técnicas estereotipadas que reproducen modelos de probada eficacia (Alarcón, Cárdenas, Miranda \& Ureña, 2009; Pinheiro, Camerino \& Sequiera, 2014). Como consecuencia, es lógico que el EAR, desde esta perspectiva, sea visto como un profesional con un perfil de profundos conoci-

Fecha recepción: 09-02-15- Fecha envío revisores: 09-02-15- Fecha de aceptación: 25-06-15 Angeles Filgueira Perez gelesfp@gmail.com mientos biomecánicos, anatómico-fisiológicos y técnicos (Moreno \& Del Villar, 2004; Lledó, Martínez \& Olmedo, 2014).

En segundo lugar, abundan los estudios que consideran al entrenador desde el enfoque pedagógico, es decir, identifican al profesor de Educación Física con la figura del entrenador en la iniciación deportiva y el deporte escolar (Añó, 1997; Ruiz \& Cabrera, 2004; Valero \& Tarifa, 2006; Sousa, Cruz, Torregrosa, Vilches \& Viladrich, 2006). Se refuerza la idea de que los entrenadores son educadores al entrenar a jóvenes en edad escolar (Álamo, Amador \& Pintos, 2002; Tabernero \& Llanos, 2002). Asimismo, se da por hecho que el entrenador es un profesor de Educación Física por la similitud en el proceso de enseñanza aprendizaje. Éste deberá dominar los ámbitos técnico, pedagógico y organizativo para asegurarse de que dicho proceso sea correcto. El ámbito técnico engloba todos los aspectos de la práctica deportiva (la preparación física, técnica, táctica, etc.). El ámbito pedagógico abarca todo lo concerniente a la enseñanza (estrategias, metodología, estilos de enseñanza, etc.) y el ámbito organizativo se relaciona con los aspectos externos de la práctica educativa-deportiva (horarios, instalaciones, organización de la competición, etc.).

El planteamiento que se hace es que el niño está empezando a formarse como deportista, así que con la metodología adecuada, unos objetivos educativos de acorde a la edad, sumados al respeto por los intereses del niño, harán que éste se ilusione por la práctica de algún deporte (Nuviala, León, Gálvez \& Fernández, 2007) . Por lo tanto, necesitará una formación psicológica y pedagógica apropiada para poder desarrollar su labor, convirtiendo así la figura del profesor de Educación Física en la más idónea. Además, cuando se enseña se transmite algo más que conocimientos (Pozo, 1999). Las connotaciones éticas de la docencia llevan consigo un ejercicio responsable, con deberes y responsabilidades por parte del que enseña (Hortal, 2014). En el deporte de alto rendimiento son pocos los estudios que profundizan sobre el papel de transmisión de valores y competencias educativas del entrenador (Pinheiro, et al., 2014).

En tercer lugar, las publicaciones de los deportes de equipo(Cubeiro \& Gallardo, 2010; García, 2006) replantean la figura del entrenador desde el enfoque del liderazgo dominante. Las investigaciones sobre las características y funciones del entrenador proceden del campo de la 
psicología y de la sociología (Gorbunov, 1988). El conocimiento de las actuaciones y actitudes derivadas del ejercicio de su profesión son investigadas principalmente por la psicología. Los estudios se centran casi en exclusiva en el rol del liderazgo (Hernández-Mendo, 2003), las habilidades sociales que manifiestan los EAR con los deportistas (García, 2007; Pérez, 2002; Ruiz, 2006) y el papel del entrenador en los deportes de equipo. La clasificación tipo del fenómeno de liderazgo considera las figura del entrenador autoritario, democrático y permisivo (Garcia, 2007; Hernández-Mendo, 2003; Leo, García-Calvo, Parejo, SánchezMiguel \& Sánchez-Oliva, 2010; Moreno \& Del Villar, 2004; Ruiz 2006; Ruiz-Risueño, 2008).Así pues, es muy complicado clasificar a un entrenador dentro de una de ellas y con unas características totalmente coincidentes con las de una tipología única. No obstante, en las investigaciones realizadas suele ocurrir que se da una identificación entre el entrenador y un modelo predeterminado (Garcia, 2007; Moreno \& Del Villar, 2004). Tal cuestión permite obtener categorías analíticas simplificadas y poner el acento sobre aquellas características más significativas y persistentes de cada profesional.

Si nos situamos en este contexto tipológico de modelo de entrenador según su estilo de dirección de equipos y personalidad, sabemos que no se corresponde con personalidades puras, de ahí que puedan mostrar rasgos correspondientes a varias categorías. Los estudios y trabajos enfocados en la formación del entrenador se centran en modalidades deportivas colectivas como son los trabajos de fútbol, balonmano, baloncesto (Feu, Ibáñez, Lorenzo, Jiménez \& Cañadas, 2012; Martínez, 2012; Leo, Sánchez-Miguel, Sánchez-Oliva, Amado \& García-Calvo, 2014; Leo, García-Calvo, Parejo, Sánchez-Miguel \& Sánchez-Oliva, 2010).

La premisa del entrenador de los deportes de equipo es que tiene que mantener al grupo de jugadores unido y lograr que trabajen en equipo para conseguir el objetivo. Se convierte en un líder al establecer la dirección del equipo y la preparación táctica prima sobre otras preparaciones.

Por tanto, existen numerosos estudios sobre la figura del entrenador en la iniciación, deporte escolar y deportes de equipo y no así de la figura del entrenador de alto rendimiento en deportes individuales, en concreto, del entrenador de atletismo. Tras profundizar en las publicaciones y en sus diversos modelos de EAR, queda por resolver la cuestión de si cada una de estas perspectivas (técnica, educativa y de liderazgo) es vista de modo aislado y hermético o es de interés estudiar la caracterización de su perfil desde una perspectiva integral y amplia.

Es difícil dar una respuesta tajante puesto que parece que solo la perspectiva técnica ha sido verdaderamente trabajada y justificada en el ámbito del alto rendimiento deportivo. Es cierto que se ha escrito mucho sobre el modelo técnico (Carreras \& Jiménez, 2010; Gonzalez, 2010; Valero, 2008; Ruiz \& Bañuelos, 1997) y que su justificación no es cuestionada en el ámbito profesional; sin embargo, parece necesario reforzar este modelo con rasgos provenientes de otros enfoques, destacando de modo significativo, el que aporta el ámbito educativo por la transmisión de valores éticos vinculados al juego limpio, el respeto a los demás, superación, etc. Debido a la importancia de conocer la propia valoración que hacen los entrenadores del entorno del alto rendimiento deportivo, los objetivos específicos de este estudio se centran en los rasgos y características que deben conformar su perfil.

\section{Material y método}

Los resultados que se presentarán en este trabajo forman parte de una investigación más amplia, llevada a cabo mediante encuesta, en la que se abarcaron tres grandes objetivos: el perfil del entrenador, el perfil del deportista y la figura del formador de entrenadores en el Practicum. La consistencia del cuestionario planteado fue supervisada por nueve expertos de cada una de las áreas estudiadas: dos expertos relacionados con las federaciones deportivas y entrenadores (ambos presidentes de federaciones autonómicas y miembros de la federación nacional), dos expertos relacionados directamente con el atletismo (ambos entrenadores y profesores universitarios y miembros de la Escuela Nacional de
Entrenadores), dos expertos en el área de Psicología (ambos docentes universitarios en ese ámbito), dos expertos en el área de Ética y Deontología profesional (ambos docentes universitarios en ese ámbito), un experto en elárea de Didáctica y Pedagogía (docente universitario en ese ámbito). A todos ellos se les pidió que revisaran una encuesta piloto, diseñada inicialmente, sobre la que hicieron aportaciones basadas en los enunciados y aclaraciones de las preguntas que fueron incorporadas al cuestionario final empleado.

Los criterios de elección de expertos se basaron en cubrir todas y cada una de las áreas que abarcaba la investigación en su conjunto. Profesionalmente, la característica común a todos ellos, era su dedicación a la docencia y a la práctica deportiva de la modalidad de atletismo de alto nivel, a excepción de las personas vinculadas al área de Ética y Deontología profesional y al área de federaciones deportivas y entrenadores.

El proceso para establecer el universo del estudio fue complejo. Se partió del listado oficial del censo electoral definitivo de entrenadores de la Real Federación Española de Atletismo (RFEA) en 2012. Dicho listado incluía a 736 personas, pero planteaba diversos problemas, ya que no distinguía entre entrenadores en activo y los que ya no lo eran, ni tampoco diferenciaba entre las categorías federativas de monitor (nivel I), entrenador de club (nivel II) y entrenador nacional (nivel III). Simplemente, figuraban en él todas las personas que habían pagado la licencia y por tanto tenían derecho a voto. Ante estas dificultades se optó por establecer unos criterios para delimitar la población objeto de estudio, que fueron consensuados con los expertos y se resumen a continuación:

a) Profesionales dedicados al entrenamiento que en el momento del estudio estuviesen en activo.

b) Entrenar o haber entrenado a deportistas de alto nivel (que hubiesen participado en los Juegos Olímpicos, en encuentros internacionales, en algún campeonato del mundo o europeo absoluto o, también, que estuviesen entre los ocho primeros del ranking absoluto de la RFEA obien hubiesen sido medallistas en categorías promesa-junior en campeonatos estatales o internacionales, como establece la normativa de la RFEA);

c) Haber entrenado a categorías inferiores y llevar ejerciendo como entrenador un mínimo diez años.

Una vez delimitado el universo del estudio (70 entrenadores), se llevó a cabo la selección de la muestra, tratando de cubrir los distintos sectores que componen el atletismo, ya que las diferentes disciplinas presentan gran variedad en sus exigencias técnicas y anatomo-fisiológicas (saltos, lanzamientos, velocidad, medio fondo y fondo, marcha y pruebas combinadas). Las principales características de la muestra se resumen en la ficha técnica siguiente (Tabla 1).

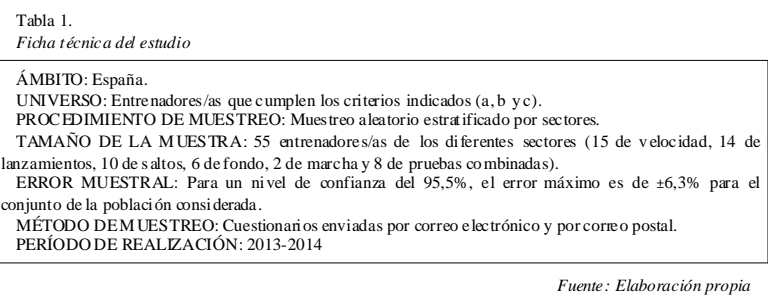

Los datos recogidos forman parte de una investigación más amplia, llevada a cabo mediante encuesta, para la que se diseñó un cuestionario de 78 preguntas en el que se abarcaban tres temas: el perfil del entrenador y del deportista, así como la figura del formador de entrenadores en el Practicum. En este trabajo nos centramos en el análisis de las cuestiones que se incluyen en el anexo y que corresponden al primer tema.

Cabe mencionar que nueve de los entrenadores encuestados desarrollan su actividad simultáneamente en varios sectores y que no quedó ninguna modalidad sin cubrir. Por otra parte, se trató de reproducir en la muestra la proporción que representa cada sector, para garantizar una adecuada representación de cada grupo.

A las personas que accedieron a colaborar en el estudio se les enviaron los cuestionarios diseñados, divididos en tres bloques temáticos. En el presente trabajo se analizarán los datos obtenidos en el primer 
bloque, relativo al perfil del entrenador, en el cual se incluían preguntas sobre los siguientes aspectos: los rasgos personales y profesionales del entrenador, sus funciones, sus conocimientos prácticos, la importancia de los valores en el desarrollo de su función, las situaciones que le son más problemáticas como entrenador, los principios éticos presentes en el mundo deportivo y el ambiente de trabajo en el entrenamiento.

Se diseñó una hoja de cálculo en formato Excel para la grabación de datos, que luego fueron depurados y analizados utilizando los programas Excel 2013 y SPSS versión 22. En particular, para el análisis de los datos relativos a las variables consideradas en este estudio, se obtuvieron dos medidas de posición: la media aritmética y la moda; en base a las cuales se realizó la clasificación de los aspectos que conformaban cada sub-bloque. Para llevar a cabo esto último, se tuvo presente que el orden debía establecerse de forma inversa según se tratase de clasificar la valoración o la priorización asignadas a cada elemento de un conjunto, de modo que los primeros puestos de la jerarquía debían corresponder a los ítems que obtuvieran las mayores puntuaciones en el caso de las valoraciones, mientras que lo sería para las menores puntuaciones si se trataba de prioridades. Concretamente, en este trabajo se estableció cada clasificación de valoraciones organizando los ítems por orden decreciente de la moda y, en caso de empate, por orden decreciente de la media. La clasificación de prioridades siguió un criterio similar, si bien considerando órdenes crecientes tanto de la moda como de la media.

\section{Resultados y discusión}

En la primera parte de esta investigación se trataba de establecer cuáles debían ser las características personales que debe poseer un entrenador para el buen desarrollo de sus funciones. Para ello, las personas encuestadas tenían que hacer una valoración en una escala de 1 a 6 (donde 1 indica nada y 6 es la máxima valoración), de diversos aspectos ligados a las características personales. La Figura 1 resume la información obtenida.

\section{Raggos personales}

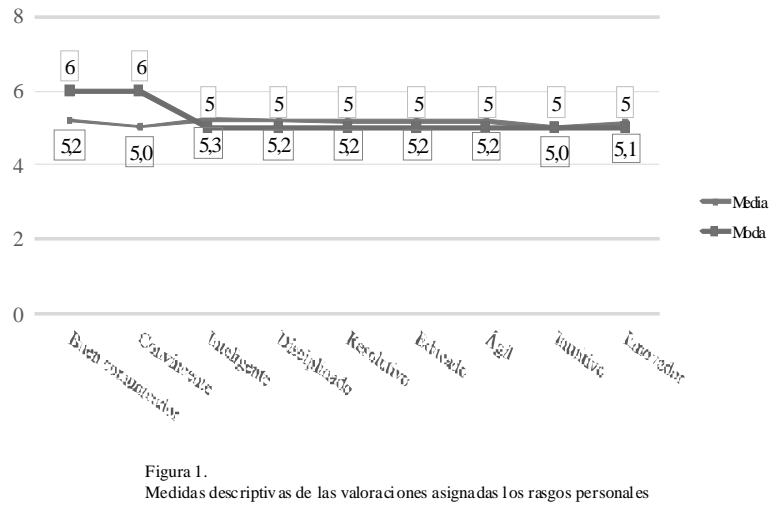

De acuerdo con la metodología descrita, la clasificación de los diferentes rasgos por orden decreciente de valoración, se corresponde con las categorías que aparecen en el eje horizontal de izquierda a derecha. Si analizamos la importancia de los rasgos que aparecen en la Figura 1 , vemos que ser un buen comunicador y ser convincente son los aspectos principales que, en su opinión, deben caracterizar al entrenador (Pérez, 2002). Es decir, el hecho de ser capaz de exponer el propio pensamiento de la mejor forma posible, así como conseguir cambiar el punto de vista de otros a través del razonamiento. Menor valoración reciben, aunque sin grandes diferencias, otros rasgos como la intuición o la innovación, a pesar de que pueden ser un activo de la capacidad de razonamiento ante la falta de información que puede haber en determinadas circunstancias.

A continuación, nos centramos en estudiar las características del perfil profesional del entrenador, cuyos resultados se presentan en la Figura 2.

De los resultados obtenidos se deduce que los principales aspectos profesionales que deben poseer los entrenadores abarcan los cono-

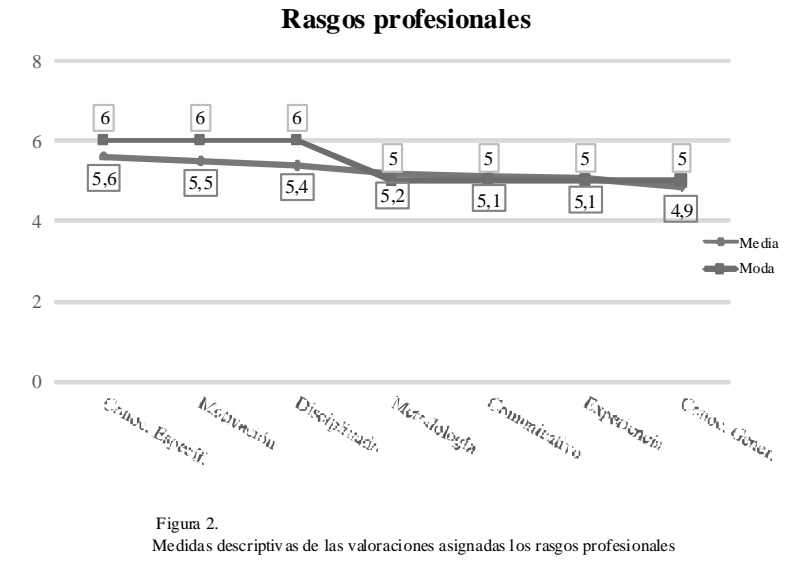

cimientos específicos del deporte de que se trate (lo que entendemos por el dominio de las técnicas y sus métodos de aprendizaje), su grado de motivación o la disciplina con la que desarrollan su actividad. Otras características de su perfil como la experiencia profesional o los conocimientos generales del ámbito deportivo-formativo han recibido una valoración ligeramente inferior. En el caso de la experiencia, los entrenadores estiman que una cantidad de años no es sinónimo de excelencia en esta labor (Ruiz \& Bañuelos, 1997). Ala vista del gráfico es así, aunque valoran la reflexión y el autoanálisis de sus propias intervenciones, así como las horas de estudio, éstas no ocupan los primeros lugares.

Las funciones que desempeña el EAR han sido también objeto de este estudio como queda reflejado en la Figura 3. En particular, se han sometido a consideración de los entrevistados actividades como programar, planificar y valorar, ya que forman parte de la secuencia de rendimiento deportivo.

Funciones del entrenador

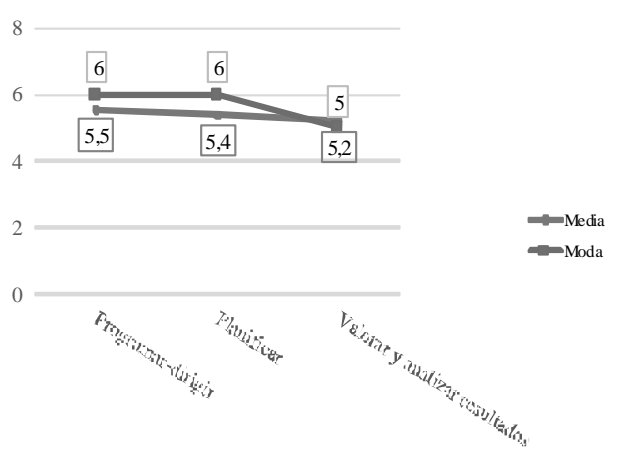

Figura 3.

Los datos obtenidos muestran que programar y dirigir se encuadran entre las funciones más valoradas. También son consideradas de importancia, aunque en menor medida, la planificación (referida a qué, a quién, para qué y de qué dispongo) y el análisis de los resultados. Esto nos indica que los entrenadores le dan más importancia al trabajo diario que al objetivo final del porqué se entrena. La programación es lo que debecentrar suatención, en el sentido de controlar la sesión oel microciclo. Lógicamente, los resultados y sus valoraciones se producen a posteriori. Es importante resaltar que en el atletismo el EAR se hace cargo de los entrenamientos y de las competiciones, abarcando la preparación completa. Al entrenador le corresponde guiar y dirigir el proceso de entrenamiento en la preparación del atleta cara a la competición y llevar a la práctica todo el potencial alcanzado en los entrenamientos, con el fin de lograr los resultados a los que se aspiran en competición (Moreno \& Del Villar, 2004; Lledó, Martínez \& Olmedo, 2014).

Por otra parte, el EAR posee un conocimiento práctico muy enriquecedor que proviene de la experiencia y la reflexión en la acción, que abarca distintos aspectos, sobre los que también se preguntó a las personas entrevistadas como puede verse en la Figura 4. 


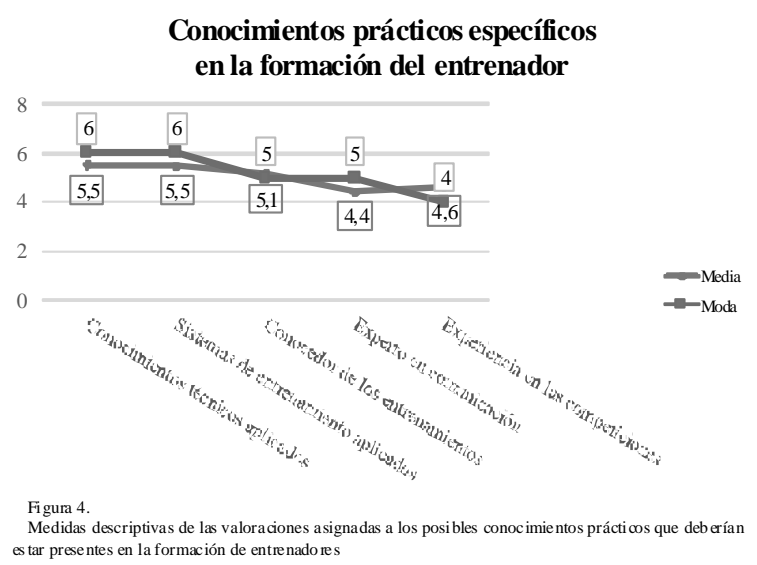

En la formación práctica específica del entrenador se ha obtenido mayor valoración en los conocimientos técnicos aplicados y los sistemas de entrenamiento aplicados. Menor valoración se asigna a la experiencia del entrenador en temas de comunicación o de participación en competiciones. Esto nos indica que los entrenadores construyen a partir de su conocimiento práctico diario el resto de las opciones. Dicho conocimiento implica saber cómo hacer algo y ser capaz de actuar, en este caso, planificar y dirigir los entrenamientos con éxito.

El EAR, como persona, posee unos valores ético-morales que manifiesta en el ejercicio de su labor deportiva. Con objeto de caracterizarlos, se sometieron a consideración de los entrevistados un rango de valores que debían ordenar según la prioridad que les asignasen. La Figura 5 que se presenta a continuación resume la información obtenida.

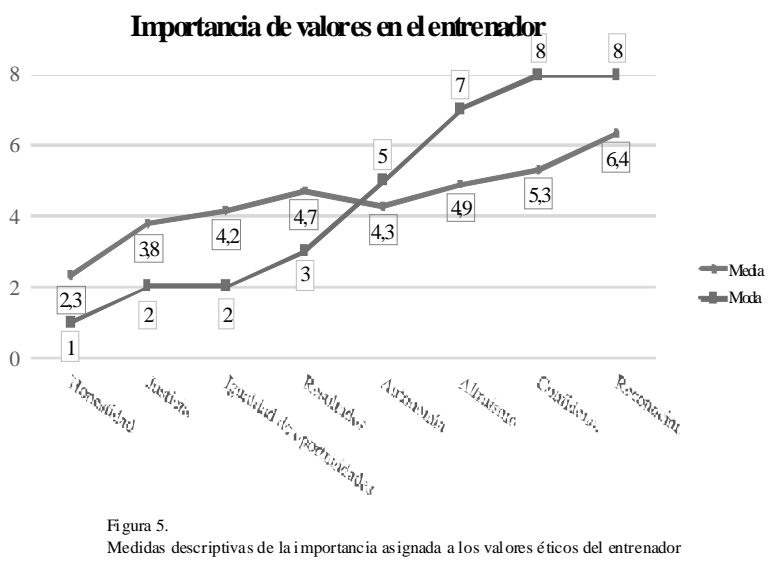

Siguiendo la metodología propuesta para el análisis de este tipo de cuestiones, la clasificación de los diferentes aspectos por orden decreciente de prioridad se corresponde con el que presentan las categorías del eje horizontal de izquierda a derecha. Teniendo esto presente, la honestidad y la justicia son los valores que consideran más importantes, seguidos por la igualdad de oportunidades. En el otro extremo se encuentran la confidencialidad o el reconocimiento. La honestidad es el primer valor y el reconocimiento el que ocupa el último lugar. La justicia y la igualdad son los valores que marcan la relación entre atletas y entrenadores, es decir, que el vínculo entrenador-atleta está marcado por la integridad y lo que menos les preocupa es el reconocimiento, en este caso, social (Ruiz, 2004).

En el contexto deportivo de alto rendimiento existen situaciones que resultan problemáticas tanto para el atleta como para el EAR. En la Figura 6 se ha tratado de establecer cuáles son las que consideran prioritarias, en el sentido de que generan mayor preocupación en los entrenadores a la hora de afrontarlas.

Los datos resultantes muestran que las tres situaciones más problemáticas a las que deben hacer frente, son, por este orden: el estilo de vida del deportista frente al deporte que practica, la relación entre la vida
Situaciones problenńticas

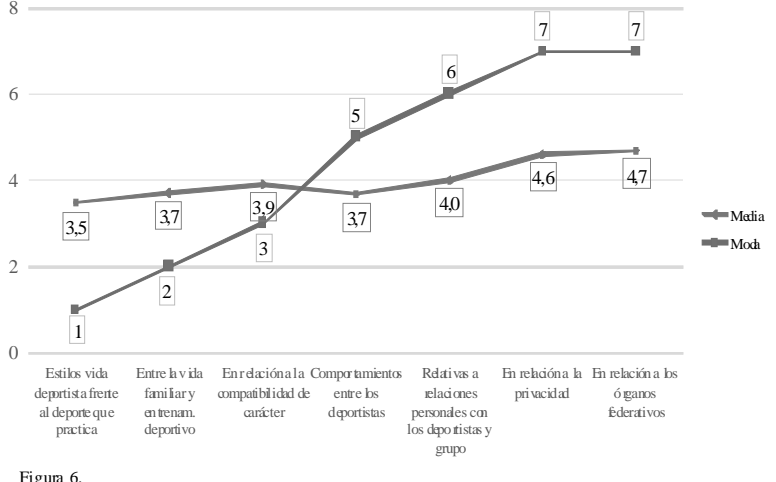

Figura 6.

familiar y el entrenamiento deportivo y la compatibilidad de caracteres. Menor preocupación generan en el entrenador las situaciones relacionadas con los órganos federativos o institucionales que tienen competencia deportiva. Estos resultados nos llevan a pensar que los entrenadores consideran prioritario «institucionalizar» la vida del deportista, es decir, eliminar las preocupaciones que afectan a su rendimiento y lo apartan de todo aquello que tenga que ver con el logro de los objetivos marcados. El estilo de vida del deportista afecta al entrenamiento denominado invisible (alimentación, descanso y horas de sueño), por lo cual, esto afectará al rendimiento (Ruiz \& Bañuelos, 1997). En el caso del entrenador de atletismo, se plantean graves dificultades entre la conciliación de la vida familiar y el entrenamiento deportivo. La dedicación y la entrega no se ve recompensada en lo material en la mayoría de los casos.

Esta forma de vida no siempre es entendible en un marco tradicional familiar. La compatibilidad de caracteres condiciona la actitud y comportamiento del entrenador y, por lo tanto, determinará la forma y grado de éxito con cada deportista (Pérez, 2002)

Ligado con los valores éticos estudiados anteriormente, la conducta del EAR se rige por unos principios que han tratado de caracterizarse en esta investigación. La Figura 7 resume la prioridad asignada en el ámbito deportivo a los cuatro principios éticos sometidos a consideración. Estos principios establecen la conducta correcta ante problemas éticos que tienen que ver con la vida en general (Hortal, 2014). La ética que tenga el entrenador condiciona y fundamenta la solución ética de cada intervención sobre el deportista. El principio de autonomía significa tener en cuenta al deportista como persona y sujeto de derecho, respetando sus decisiones y convicciones. El principio de justicia supone tratar a cada uno como corresponda y promover la defensa ante las injusticias en el ámbito específico del deporte y social. El principio de beneficencia significa hacer bien una actividad y hacer el bien a otros mediante una actividad bien hecha. Supone que el entrenador posee una formación y conocimientos de los que el deportista carece, por lo que el entrenador sabe y por tanto, decide lo más conveniente para el deportista. El principio de no maleficencia significa abstenerse intencionadamente de realizar acciones que puedan causar daños o perjudicar a otros.

De la Figura 7 se desprende que los entrenadores priorizan el

\section{Principios éticos presentes en el mundo deportivo segun el entrenador}

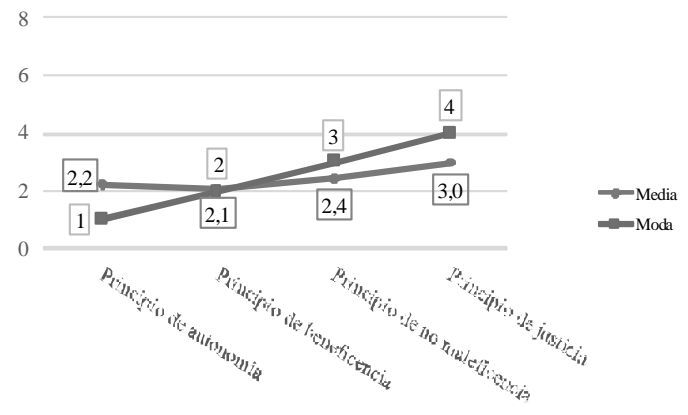

Fi gura 7.
Medidas descri ptivas de las valoraciones asignadas a los principios eticos 
principio de autonomía sobre los demás, incluido el de justicia o el de no maleficencia (ante todo no hacer daño). Estos resultados se alejaron de lo esperado, si se tiene presente que la justicia ocupa la segunda posición en su escala de valores (Figura 5) o el hecho de que las lesiones suelen constituir una de las principales preocupaciones de los entrenadores. La relación entrenador-atleta se basa fundamentalmente en los principios de no maleficencia y beneficencia, pero cuando estos principios entran en conflicto, es el principio de justicia el que entra en juego para mediar entre ellos, teniendo muy en cuenta el principio de autonomía (Hortal, 2002).

Por último, un factor fundamental que se trató en este estudio fue la caracterización del ambiente de trabajo en el entrenamiento, por la repercusión que ello tiene en el rendimiento deportivo véase Figura 8.

\section{Ambiente de trabajo en el entrenamiento}

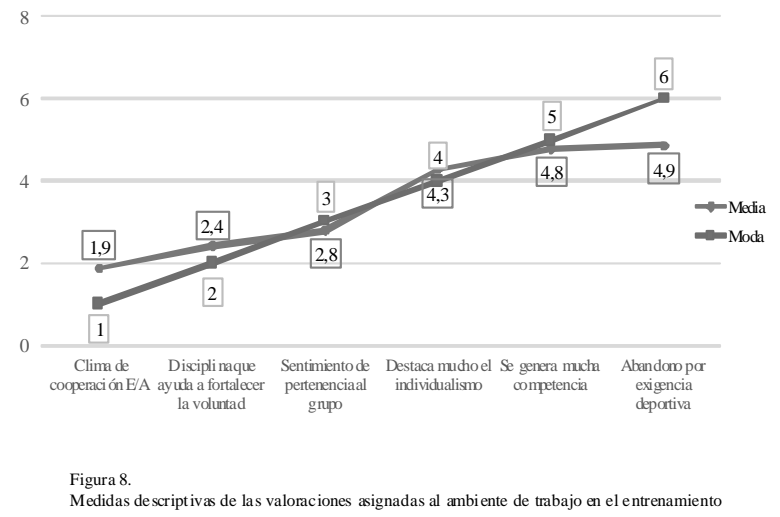

Los entrenadores consideran prioritario propiciar en el entrenamiento un clima de cooperación y apoyo mutuo entre los deportistas y el entrenador, conjuntamente con la aplicación de una disciplina de trabajo que ayude a fortalecer la voluntad del deportista (Gorbunov, 1988). Esto es entendible ya que en un contexto institucionalizado, el hecho de que un deportista abandone depende del entrenador. También es evidente que, en el binomio entrenador-atleta, la comunicación y la compatibilidad de caracteres es fundamental para facilitar la consecución de resultados (Pérez, 2002). Se supone que el entrenador crea una atmósfera positiva mostrando una preocupación e interés genuino por sus deportistas.

\section{Conclusiones}

Las conclusiones más destacadas de este estudio sobre la caracterización del perfil del entrenador de alto rendimiento en atletismo se resumen a continuación:

- Desde la perspectiva de los entrenadores, en los rasgos personales destacan la importancia de ser un buen comunicador y convincente.

- En el aspecto profesional, los entrenadores deben tener un gran bagaje de conocimientos específicos del deporte (las técnicas y sus métodos de enseñanza-aprendizaje), pero también deben poseer un alto grado de motivación para desarrollar este trabajo.

- En su ética profesional deben predominar los principios de autonomía y beneficencia, de modo que en el desarrollo de sus funciones primen valores como la honestidad y la justicia.

- El ambiente de trabajo en el entrenamiento debe ser favorablea que se cree un clima de cooperación y de apoyo al deportista. No obstante, los entrenadores deben aprender a afrontar situaciones problemáticas a lo largo de su carrera, relativas principalmente a los estilos de vida de los deportistas frente al deporte que practican o la conciliación familiar con el entrenamiento deportivo.

En resumen, los resultados nos revelan que el entrenador se percibe a sí mismo como un profesional polifacético que necesita dominar aspectos técnicos, educativos y de liderazgo. Los primeros conllevan el control de las estrategias técnico-metodológicas orientadas a la consecu- ción de resultados eficaces y eficientes, tanto en el proceso de entrenamiento como en el desarrollo de las competiciones. Los aspectos educativos ayudan a formar al deportista como ser social. También destacan los entrenadores la importancia de la tarea del entrenador-líder, carismático, en quien se deposita la confianza con el fin de obtener los mejores resultados.

Esto abre el camino a futuros estudios sobre las competencias educativas, basadas en la transmisión de los valores del deporte de alto rendimiento y sobre la posibilidad de plantear nuevos enfoques relacionados con el papel del entrenador de atletismo y los problemas que quedan por resolver relacionados con el marco profesional de esta figura.

\section{Agradecimientos}

Este trabajo no sería posible sin la colaboración prestada por los entrenadores, que han participado con gran interés y esfuerzo en esta investigación. La autora agradece también el asesoramiento de la profesora Pilar García Soidán y los comentarios recibidos por parte del editor y los revisores, que han contribuido a mejorar el contenido de este trabajo.

\section{Referencias}

Alamo, J.M., Amador, F., \& Pintos, P. (2002). Función social del deporte escolar. El entrenador del deporte escolar. Educación físicay deportes (45). Recuperado de http://www.efdeportes.com

Alarcón, F; Cárdenas, D; Miranda, M.T.; \& Ureña, N. (2009).Los modelos de enseñanza utilizados en los deportes colectivos. Investigación educativa, (23)13, 101-128.

Año, V.(1997). Planificacióny organización del entrenamiento juvenil. Gymnos. Madrid.

Calero Morales, S.C. (2011). Variables significativamente influyentes en el rendimiento del pasador de voleibol. Revista Internacional de Medicina y Ciencias de la Actividad Física y el Deporte, (42)11, 347-361.

Carreras, J., \& Jiménez, J (2010). Metodología de enseñanza utilizada en la enseñanza del tenis dentro de la etapa de iniciación. Retos, (18) 60-65.

Cubeiro, J.C., \& Gallardo, L. (2010). Liderazgo Guardiola. Alienta. Barcelona.

Del Villar, F., \& Fuentes, J.P. (1999). Las destrezas docentes en la enseñanza del tenis.

En Fuentes, J.P., Cervelló, E.M., Del Villar, F., Gusi, N., \& Moreno, F.J. Enseñanza y entrenamiento del tenis. Fundamentos didácticos y científicos. Servicio de publicaciones. Universidad de Extremadura.

Feu, S., Ibáñez, J., Lorenzo, A; Jiménez, S., \& Cañadas, M. (2012).El conocimiento profesional adquirido por el entrenador de balonmano: experiencias y formación. Psicología del Deporte, (1)21,107-115.

García, J.A. (2006). Liderar y entrenar un equipo de balonmano: de la dirección del grupo a la planificación técnico-táctica. Wanceulen. Sevilla.

García, F.E. (2007). El rol del entrenador. Asociación de Psicología del Deporte Argentina, 1-32.

González, G(2010).¿Quéimportancia tiene la función psicopedagógica del técnico deportivo para la mejora de los jugadores en un club? Retos, (18), 35-40.

Gorbunov, GD. (1988). Psicología del deporte. Moscú.

Hernández-Mendo, A., \& Canto, J. M. (2003). El liderazgo en los grupos deportivos. En Hernández-Mendo, A. (Coord.), Psicología del deporte (vol. I): Fundamentos 2 (pp. 6-28).Wanceulen. Sevilla.

Hortal, A (2002). Ética general de las profesiones. Desclee. Bilbao

Ibañez, S.G., \& Medina, J (1999). Relaciones entre la formación del entrenador deportivo y la formación del profesor de educación física. Apunts, (56)39-45.

Leo, F.M., Sánchez-Miguel, P.A., Sánchez-Oliva, D., Amado, D., \& 
García-Calvo, T. (2014).Análisis de los procesos grupales y el rendimiento en fútbol semiprofesional. Revista Internacional de Medicina y Ciencias de la Actividad Física y el Deporte, (53)14, 153-168.

Leo, F.M., García, T., Parejo, I., Sánchez, P.A., \& Sánchez, D. (2010). Interacción de la cohesión en la eficacia percibida, las expectativas de éxito y el rendimiento en equipos de baloncesto. Psicología del Deporte, (1)19, 1-14.

Lledó, E., Martínez, G., \& Huertas, F. (2014). El perfil del entrenador de fútbol en la etapa escolar en escuelas de clubes deélite de la Comunitat Valenciana. Cultura, cienciaydeporte, (25)9, 57-68.

Martínez, J.A. (2012). Entrenador nuevo, ¿victoria segura?. Revista Internacional de Medicina y Ciencias de la Actividad Física y el Deporte, (48)12, 663-679.

Moreno, M.P., \& Del Villar Álvarez, F. (2004). El entrenador. Manual práctico para su desarrollo. Inde. Barcelona.

Nuviala, A., León, J.A., Gálvez, J., \& Fernández, A. (2007). Qué actividades deportivas escolares queremos. Qué técnicos tenemos. Revista Internacional de Medicina y Ciencias de la Actividad Física y el Deporte, (25)7, 1-9.

Ortega, E.(2005). Autoeficaciay deporte. Wanceulen. Sevilla.

Pérez, M.C. (2002). Estudio cualitativo sobre entrenadores de alto rendimiento deportivo. Psicología del deporte, (1)11, 9-33.

Pinheiro, V., Camerino, O., \& Sequiera, P. (2014). El fair play en la iniciación deportiva, un estudio con entrenadores de fútbol. Retos, (25), 3235.

Pozo, J.I (1999). Aprendices y maestros: la nueva cultura del aprendizaje. Alianza. Madrid.

Ruiz, G., \& Cabrera Suarez, D. (2004).Los valores en el deporte. Revista de Educación, (335), 9-19.

Ruiz, L.M., \& Sánchez, F. (1997). Rendimiento deportivo: Claves para la optimización de los aprendizajes. Gymnos. Madrid.

Ruiz, R(2006). Diferencias de liderazgo en entrenadores de judo a nivel competitivo. Cuadernos de psicología del deporte, (2)6, 21-38.

Ruiz-Rioseco, J. (2008). El papel del entrenadoren la capacidaddecisional de los jugadores. Retos, (13),5-10.

Sousa, C., Cruz, J., Torregrosa, M., Vilches, D., \& Viladrich, C. (2006). Evaluación conductual y programa de asesoramiento personalizado a entrenadores (pape) de deportistas jóvenes. Revista de Psicología del Deporte, (2)15, 263-278.

Saneugenio,A., \& Escontrela., R. (2000) EImodelo critico-reflexivo y el modelotécnico: sus fundamentos y efectos en la formación del docente de la

RASGOS PER SONALES

Otros:

RASGOS PROFESIONALES personas) profesional)

Otros:

Experto en comunic ación

Otros:

Confidencialidad

Resultados

Otmos

Otros: educación superior. Docencia Universitaria, (1)1, 11-39.

Tabernero, B., Márquez, S., \& Llanos, C. (2002). Elementos a analizar en el proceso de iniciación deportiva. Retos, (1), 9-15.

Valero, A., \& Tarifa Pérez, J. (2006). Taxonomía de las disciplinas atléticas en función del grado de dificultad para su enseñanza en la iniciación al atletismo. Habilidad motriz (27), 58-65.

Valero, A (2008). Funciones y responsabilidades del profesor y los alumnos en el modelo de enseñanza ludotecnico de iniciación a la práctica de atletismo. Retos, (13), 15-18.

\section{Anexo- cuestionario}

¿Cuáles son las caracteństicas o rasgos personales y profesionales que debe p oseer un buen profesional (entrenador) en el ámbito educ ativo dep ortivo? 1: nada, 2: poco, 3: regular, 4: bastante, 5: mucho 6: muchísimo.

Inteligente (capacidad de aprender y adaptarse al medio)

Agilidad de anál isis ante situaciones complicadas

Convincente (el que es capaz de cambiar el punto de vista de otros mediante el razonamiento)

Buen comunicador (es ser capaz de exponer el propio pensamiento de la mejor forma posible)

Disciplinado (cumplir lo previsto)

Educado (respet o hacia los demás)

Resolutivo (capacidad de solucionar di stint as situaciones)

Intui tivo ( tener la información previa sin haber hecho la secuencia de anál isis lógica)

Innovador (actualizar s us conocimi entos teórico-prácticos)

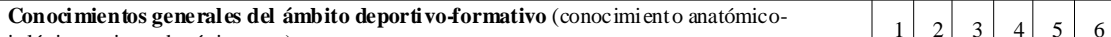
fisiológico, psicopedagógico, etc.)

Conocimien tos específicos del deporte

( dominio/s de las técnica/s y sus métodos de aprendizaje)

Disciplinado (respons abilidad en su trabajo)

Comunicativo (es sercapaz de exponer el propio pensamiento de

\begin{tabular}{llllllll}
1 & 2 & 3 & 4 & 5 & 6 \\
\hline & 1 & 2 & 3 & 4 & 5 & 6 \\
\hline
\end{tabular}

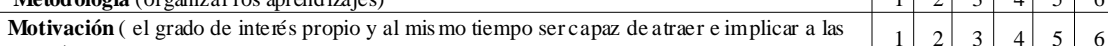

Experiencia (cúmulo de errores/ aciertos y las concl usiones extraídas aplica das en la vida

¿Cuáles son las funciones del entrenador?

Planificar (referida a qué a quien, para qué, de qué dispongo)

\begin{tabular}{l|l|l|l|l|l|l|} 
& 1 & 2 & 3 & 4 & 5 & 6 \\
\hline
\end{tabular}

Programar-dirigir (control de la planificación para poder modific ar en b ase a las

competiciones, lesiones, estudios, clima.)

Valoración y análisis de resul tados

¿Qué conocim ientos Prácticos- Específicos deberían estar incluidos en la Formación de los entrenadores? Con ocimi ent os técnicos aplic ados

Sistemas de entrenamiento aplicados

Experiencia en las competiciones

Con ocedor de los entrenamientos

Ordena los siguientes valores en relación a la importancia que tiene para ti en el desarollo de tu función como

entrenador (ordena por orden de prioridad de 1 a 8):

Justicia(trato igual a los igual es y desigual a los desiguales, atendiendo a las circunstancias de cada uno)

Igual dad de oportunidades(los que están en las mismas circunstancias reciben el mismo trato)

Altruismo (actuar sin buscar in tereses)

Autonomía ( hacerse cargo de mis decisiones y responsabilizarse de las consecuencias)

Reconocimiento (de la labor que haces en favor de la sociedad)

Honestidad (integri dad en el obrar)

Qué situaciones como entrena dor te han creado problemas? Ordenar por prioridad de 1 a 7.

Relativas a las rel aciones personales con los deportist as y el grupo

Comportamientos entre los deportistas

En relación a la compatibilidad de carácte

En relación a la privacidad (todo aquello que esta fuera del es pacio depo rtivo)

Entre la vida fami liar y entrenamiento deportivo

En relación a los órganos federativos o institucional que tienen competencia deportiva

Estilos de vida del dep ortista frente al deporte que practica

¿Cuál de los siguientes principios éticos crees que está más presente en el mundo del deporte en el que desarrollas tu

labor como entrenador? Ordena por orden de prioridad de 1 a 4 .

Principio de ben eficencia: hacer bien una actividad y hacer el bien a ot ros mediante una actividad bien hecha.

Principio de autonomía: en el ámbito del deporte el principio de autonomía signi fica tener en cuenta al deportista como persona y sujeto de derecho; respect ando sus convicciones y decisiones.

Principio de justicia: en el ámbito del deporte se traduce en promover el compromi so de los profesionales con las

injusticias tanto del ámbito específico del deporte como con la defensa y apovo de las causas porla jus ticia social.

Principio de no maleficencia: ante todo no hacer daño.

Cómo defines el ambiente de trabajo en tu ejercicio como entrenador? establece el orden de priorid ad de 1 a 6 .

Existe un clima de cooperación y apoyo mutuo entre deportis ta-entrenador

Se genera mucha competencia en el entrenami ento y stress en los deportistas

Existe una disciplina que avuda a fortalecer la voluntad de los deportistas

Es tanta la exigencia deportiva que muchos deportistas abandonan

Es priori tario el sentimien to de pertenencia al grupo

Destaca mucho el individualismo 